\title{
Conocimiento, percepciones y usos del Crocodylus moreletti en la Reserva de la Biosfera Pantanos de Centla
}

\section{Knowledge, perceptions and uses of Crocodylus moreletii in Reserva de la Biosfera Pantanos de Centla}

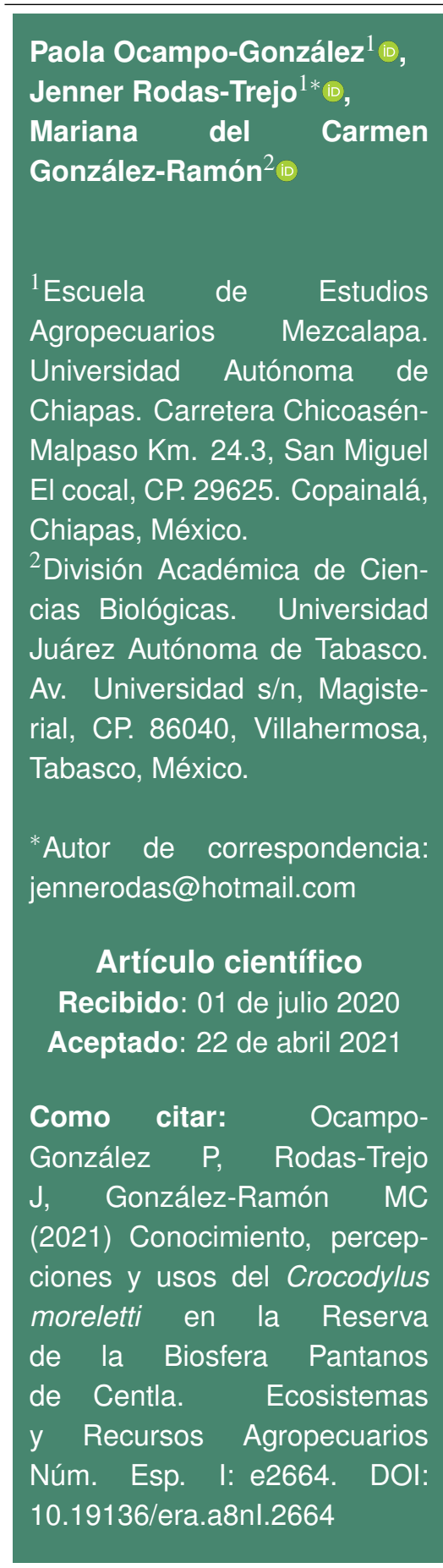

RESUMEN. La Reserva de la Biosfera Pantanos de Centla (RBPC) representa uno de los principales sitios de refugio, reproducción y alimentación del cocodrilo de pantano (Crocodylus moreletii) en México. El objetivo del trabajo es identificar el conocimiento, percepción, interacciones, usos y aprovechamientos que pobladores de la RBPC tienen hacia la especie. Entre los meses de agosto a octubre 2018 se realizaron entrevistas semiestructuradas a adultos y talleres de educación ambiental con niños en cuatro localidades de la Reserva. Mediante las entrevistas se identificó un notable conocimiento sobre el cocodrilo de pantano, ya que las personas interactúan constantemente con la especie durante actividades como la pesca, demostrando interés y respeto hacia ella. El uso y aprovechamiento de la especie se presenta en todas las localidades y es principalmente como alimento y medicina. EI principal factor que preocupa a los pobladores en la conservación de la especie es la contaminación de los ríos y lagunas con sustancias derivadas de la industria petrolera y actividades agropecuarias. Las actividades con niños demostraron el desconocimiento que tienen respecto a los beneficios ecológicos del cocodrilo de pantano, además de demostrar estar a favor de la cacería de diversas especies silvestres. Se considera necesario reforzar acciones para la conservación de fauna silvestre tanto en niños como adultos, ya que su consumo es una actividad común dentro de la Reserva, además de realizar más estudios que identifiquen la presencia de sustancias contaminantes tanto en los animales como en el agua y promover la difusión de los resultados.

Palabras clave: Aprovechamiento, conflicto hombre-cocodrilo, consumo, educación ambiental, interacciones.

ABSTRACT. The Pantanos of Centla Biosphere Reserve (PCBR) represent one of the main refuge, reproduction and feeding sites for the Morelet's Crocodile (Crocodylus moreletii) in Mexico. The objective of the work is to identify the knowledge, perception, interactions, uses and benefits that inhabitants of the RBPC have towards the species. Between August and October 2018, semi-structured interviews with adults and environmental education workshops with children were carried out in four locations of the Reserve. Through the interviews, a remarkable knowledge about the Morelet's Crocodile was identified, since people constantly interact with the species during activities such as fishing, showing interest and respect towards it. The use and exploitation of the specie occurs in all localities and is mainly as food and medicine. The main factor that concerns the inhabitants in the conservation of the species is the contamination of rivers and lagoons with substances derived from the oil industry and agricultural activities. The activities with children demonstrated their ignorance regarding the ecological benefits of the Morelet's Crocodile, as well as demonstrating that they are in favor of hunting various wild species. It is necessary to reinforce actions for the conservation of wildlife both in children and adults, since its consumption is a common activity within the Reserve, in addition to carrying out more studies that identify the presence of polluting substances in both animals and water and promote the dissemination of the results. Key words: Use, crocodile-man conflict, consumption, environmental education, interactions. 


\section{INTRODUCCIÓN}

Desde tiempos antiguos, diversas culturas como la egipcia, náhuatl y maya tenían una relación de respeto y admiración hacia los cocodrilos (Sigler y Gallegos 2017, Lorente 2018). En la actualidad, continúan siendo parte fundamental de las creencias, mitos y cosmogonías de distintos grupos étnicos (Cupul-Magaña 2003, García-Grajales y Buenrostro 2015), en diversas regiones son apreciados como alimento y medicina (Romeu 1998, Arias 2007, Lorente 2018), además de representar un valor económico principalmente por la comercialización de la piel y en época reciente por el ecoturismo (Valdelomar et al. 2012, Mandujano-Camacho, 2014). De manera contrastante, en algunos lugares se tiene una percepción negativa hacia ellos, ya que se considera una especie peligrosa debido a ataques a personas y ganado (García-Grajales 2013, PeñaMondragón et al. 2013), además dañina por competencia alimentaria (pesca y fauna doméstica) y espacial (hábitat) (Balaguera-Reina y González-Maya 2010). En México se han realizado diferentes trabajos para conocer las percepciones sociales, conflictos humano-cocodrilo, conocimiento ecológico y uso que poblaciones humanas hacen sobre los cocodrilos (Cedillo-Leal et al. 2011, Peña-Mondragon et al. 2013, Rodas-Trejo et al. 2018).

La Reserva de la Biosfera Pantanos de Centla (RBPC) es uno de los principales sitios de refugio, reproducción y alimentación del cocodrilo de pantano (Crocodylus moreletii) en México. Sin embargo, se desconoce la relación que pobladores locales tienen con esta especie, por lo que el objetivo del presente trabajo es identificar el conocimiento, percepción, interacciones, usos y aprovechamientos que pobladores de cuatro localidades de la RBPC tienen hacia el cocodrilo de pantano (Crocodylus moreletii), a través de la información obtenida se plantearán estrategias que contribuyan a su conservación.

\section{MATERIALES Y MÉTODOS}

\section{Área de estudio}

El trabajo se realizó en las localidades La Mixteca Segunda, Lázaro Cárdenas (El Guao), Tabasquillo y Tembladeras, pertenecientes al municipio de Centla, dentro de la Reserva de la Biosfera Pantanos de Centla (RBPC), localizada al noreste del estado de Tabasco (Figura 1). De acuerdo con datos del INEGI (2015), el número de habitantes entre las cuatro localidades es de 794 y el grado de marginación va de alto a muy alto. La RBPC es considerada uno de los humedales más importantes de Mesoamérica, es sitio NAWCA desde 1989 y humedal de importancia internacional RAMSAR desde 1995. Pertenece a la región hidrológica Grijalva-Usumacinta y abarca parte de tres cuencas: Usumacinta al norte y centro de la Reserva, Laguna de Términos al este y río Grijalva-Villahermosa al sur y oeste. Dentro de la Reserva se presentan ecosistemas como selva mediana subperennifolia y baja subperennifolia, manglar, matorral, palmares y en una mayor extensión de territorio vegetación hidrófila (Barba et al. 2014). Las actividades socioeconómicas son la pesca, agricultura y ganadería, sus habitantes son provenientes de las etnias mayas y chontales con un profundo y ancestral conocimiento del entorno, cuya economía se basa en la extracción de recursos naturales (Romero et al. 2000).

\section{Metodología}

Entre los meses de agosto a octubre 2018 se realizaron entrevistas semiestructuradas a adultos y talleres de educación ambiental con niños para identificar el conocimiento, percepción, interacciones, usos y aprovechamientos hacia el cocodrilo de pantano (Crocodylus moreletii) dentro de la RBPC.

\section{Entrevistas}

Se diseñó un cuestionario para realizar entrevistas semi-estructuradas que abordaban aspectos sobre el conocimiento, percepción, interacciones, usos y aprovechamiento que pobladores que viven dentro de la RBPC tienen sobre el cocodrilo de pantano (Crocodylus moreletii). Las entrevistas fueron 


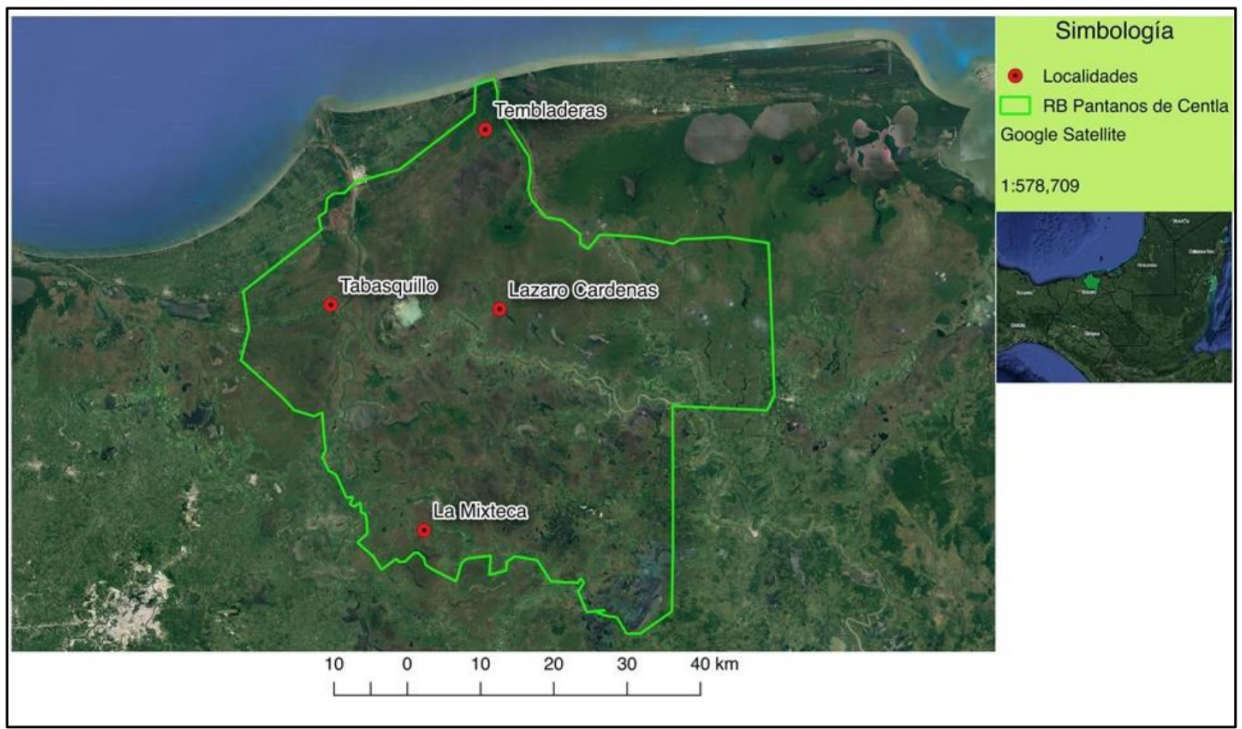

Figura 1. Localización de las localidades de estudio dentro de la Reserva de la Biosfera Pantanos de Centla. Escala: 1:578,709.

dirigidas hacia informantes clave (Peña-Mondragón et al. 2013, Albuquerque et al. 2014) seleccionados a través de la técnica de bola de nieve (Bernard 1996), en la cual se identifican a los individuos que poseen la información sobre el tema (pescadores, habitantes de márgenes del rio), respetando la identidad cultural local, y estableciendo una relación de confianza entre el investigador y los informantes. Los criterios de inclusión fueron ser mayor de edad y tener más de 20 años viviendo en la localidad. Los temas abordados en la entrevista se presentan en la tabla 1.

\section{Talleres de educación ambiental}

Se realizaron tres talleres de educación ambiental en las escuelas primarias multigrados de las localidades Tabasquillo, Lázaro Cárdenas y Tembladeras. Estuvieron dirigidos a niños de $1^{\circ}$ a $6^{\circ}$ grado de educación primaria, bajo el consentimiento informado de sus padres y con el apoyo del director de la escuela y los profesores de grupo, tuvieron una duración de tres horas en una sola sesión y consistieron en actividades de información y sensibilización ambiental sobre la importancia biológica, ecológica y cultural del cocodrilo de pantano. Las actividades realizadas durante los talleres se encuentran resumidas en la Tabla 2.

\section{RESULTADOS}

\section{Entrevistas}

Se realizaron 40 entrevistas a informantes claves de la RBPC, en las que se obtuvo información sobre el conocimiento, percepción, interacciones, usos y aprovechamiento hacia el cocodrilo de pantano (Crocodylus moreletii). El $72.5 \%$ de los entrevistados $(n=29)$ fueron del género masculino y el $27.5 \%$ $(n=11)$ del género femenino. La edad promedio de los participantes fue de $38.4 \pm 5.7$ años, dentro de un rango de 22 a 72 años. El 100\% de los entrevistados $(n=40)$ vive en alguna localidad dentro de la RBPC, el $87.5 \%(n=35)$ de los entrevistados realiza actividades que implican una relación cercana con ríos y/o lagunas (pescadores, guía turístico, lanchero).

\section{Conocimiento}

El $100 \%$ de los entrevistados $(n=40)$ conoce al cocodrilo de pantano y lo identifica como la única especie en la región. Los nombres comunes que utilizan para referirse a esta especie son cocodrilo, cocodrilo del pantano y lagarto. Las principales características físicas con las que los describen son: animales grandes, mayormente de color negro, aunque también indican que son de color amarillo y de piel 


\begin{tabular}{|c|c|}
\hline Sección & Temática \\
\hline Conocimiento & $\begin{array}{l}\text { - Especies que habitan en la región } \\
\text { - Características físicas y etológicas } \\
\text { - Hábitat } \\
\text { - Alimentación } \\
\text { - Reproducción }\end{array}$ \\
\hline Interacciones & $\begin{array}{l}\text { - Frecuencia de observación e interacción } \\
\text { - Conflicto hombre-cocodrilo }\end{array}$ \\
\hline Usos y aprovechamiento & $\begin{array}{l}\text { - Cacería } \\
\text { - Usos } \\
\text { - Aprovechamiento }\end{array}$ \\
\hline Percepción & $\begin{array}{l}\text {-Palabras a las que asocia el término "cocodrilo" } \\
\text { - Comparación del tamaño de la población entre la actualidad y diez años antes } \\
\text { - Importancia ecológica } \\
\text { - Factores que afectan las poblaciones de cocodrilo }\end{array}$ \\
\hline
\end{tabular}

Tabla 2. Actividades y objetivos planteados en los talleres de educación ambiental con niños.

\begin{tabular}{|c|c|}
\hline Actividad & Objetivo \\
\hline Presentación & Generar una relación de confianza inicial entre los facilitadores y los niños. \\
\hline Dibujo diagnóstico & $\begin{array}{l}\text { Identificar el conocimiento y la percepción que los niños tienen sobre el cocodrilo } \\
\text { de pantano, a través de la técnica de dibujo. }\end{array}$ \\
\hline Plática informativa & $\begin{array}{l}\text { Informar a los niños la importancia ecológica del cocodrilo de pantano, las ame- } \\
\text { nazas a que se enfrentan sus poblaciones como el consumo ilegal, la cacería } \\
\text { furtiva, comercialización, contaminación y degradación de su hábitat, entre otros } \\
\text { factores. }\end{array}$ \\
\hline Intercambio de ideas & $\begin{array}{l}\text { Identificar la percepción y experiencias que los niños tienen con el cocodrilo de } \\
\text { pantano. Discutir sobre aspectos de la vida diaria en que se relacionan con los } \\
\text { cocodrilos, ya sea a nivel individual, familiar y de la comunidad. }\end{array}$ \\
\hline Juegos de mesa y al aire libre & $\begin{array}{l}\text { Reforzar de manera positiva el reconocimiento de especies animales que viven } \\
\text { en su comunidad. }\end{array}$ \\
\hline Obra de teatro con títeres & $\begin{array}{l}\text { Sensibilizar a los niños sobre la importancia ecológica del cocodrilo de pantano, } \\
\text { así como de otras especies de fauna locales. }\end{array}$ \\
\hline Reflexiones finales y cierre del taller & $\begin{array}{l}\text { Responder a las dudas e inquietudes que los niños tuvieran al finalizar el taller. } \\
\text { Reflexionar sobre la importancia de las actividades que contribuyen a la conser- } \\
\text { vación de especies silvestres locales, y en específico, del cocodrilo de pantano. }\end{array}$ \\
\hline
\end{tabular}

escamosa. Los entrevistados mencionaron que el tamaño de los cocodrilos que han visto va desde los $25 \mathrm{~cm}$ hasta los cinco metros, siendo de uno a cuatro metros las medidas que mencionaron con mayor frecuencia $(70 \%, \mathrm{n}=28)$. Las características etológicas de la especie que reconocen es que son animales agresivos y territoriales. Respecto al conocimiento que se tiene sobre el lugar donde habitan, el 95\% (n = 38) mencionó que viven en el río, el $87.5 \%(n=35)$ en la laguna y el $72.5 \%(n=29)$ en el pantano. En cuanto a la alimentación del cocodrilo de pantano, el $90 \%(n=36)$ mencionó que se alimenta de peces, el $25 \%(n=10)$ de aves, el 10\% $(n=4)$ de mamíferos, $7.5 \%(n=3)$ de carroña, el $5 \%(n=2)$ de reptiles y el $2.5 \%(n=1)$ de plantas. Referente al conocimiento que las personas tienen sobre la reproducción de la especie, indicaron que el macho es más grande que la hembra, el $80 \%(n=32)$ indican que la temporada reproductiva es en la época de seca y que es raro encontrar nidos, ya que éstos los hacen en lugares alejados e internados en el pantano o el río, en lugares inaccesibles para las personas, estrategia que utilizan para proteger a sus crías.

\section{Interacciones}

El $100 \%(n=40)$ de los entrevistados mencionó que las interacciones con el cocodrilo de pantano son frecuentes, ya sea al observarlo cuando van a pescar, al cazarlo o porque lo han comido. En las localidades de Tabasquillo y Lázaro Cárdenas los pescadores y lancheros identifican a individuos que en ciertas temporadas los observan casi a diario y a quienes incluso les han asignado nombre; un ejemplo es el cocodrilo llamado Mocho en la localidad 
de Tabasquillo. El total de los entrevistados ( $\mathrm{n}=$ 40) mencionó que en su localidad no se han presentado ataques de cocodrilos a personas; sin embargo, algunos mencionaron haber escuchado de algún ataque en otra localidad dentro de la Reserva, pero no estaban completamente informados sobre lo ocurrido. En cuanto a la existencia de conflicto hombre-cocodrilo, el 100\% ( $n=40)$ mencionó que no consideran que exista, ya que no han tenido problemas de ataques ni a personas ni a sus animales, y aunque los consideran animales peligrosos, saben que no deben molestarlos para evitar inconvenientes. En la localidad de Tabasquillo, los entrevistados mencionaron que lejos de existir un conflicto hombre-cocodrilo, ellos ven una oportunidad para incrementar el turismo en la zona y con ello sus ingresos económicos, ya que ofrecen recorridos en lancha donde uno de los principales atractivos turísticos es el avistamiento de estos animales, además que en ocasiones pueden hacer uso de ellos como alimento.

\section{Usos y aprovechamiento}

El $100 \%(n=40)$ de los entrevistados no considera que la cacería de cocodrilo de pantano sea una actividad frecuente, y tampoco que se haga un aprovechamiento de esta especie, ya que el único producto del que podrían tener un ingreso económico directo es la venta de su piel. Aunque hace 20 años era una actividad común, actualmente, debido a las restricciones legales que existen, se las pagan a un precio muy económico que no es redituable ( $\$ 100$ por un adulto de dos metros de largo) por lo que es una actividad que no realizan, ya que el riesgo de cazarlo es muy grande. Sin embargo, el $35 \%(n=14)$ de las personas reconocieron en algún momento haber capturado algún cocodrilo, con fines alimenticios o medicinales. Respecto a su uso como alimento, el $37.5 \%(n=15)$ de las personas mencionaron que consumen mínimamente una vez por mes la carne de cocodrilo, principalmente la cola, ya que es la parte con mejor sabor y mayor carne. El $66.66 \%$ de ellos, mencionó que la forma en como cocinan la carne es frita, el $50 \%$ asada y el $33.33 \%$ mencionó prepararla guisada y en tamales.

El 100\% ( $n=40)$ de los entrevistados aseguró que el cocodrilo de pantano tiene propiedades curativas contra enfermedades respiratorias, entre ellas el asma, tos crónica y bronquitis. La parte del cocodrilo que utilizan para hacer el remedio curativo es la grasa de la cola en forma de jarabe, para ello calientan la grasa hasta que esté líquida y la puedan almacenar en un frasco para administrarla vía oral en forma de jarabe, ofreciendo diariamente una cucharada del remedio hasta que la persona se recupera. Hay que destacar que todas las personas consideran que es un remedio efectivo tanto en niños como adultos, y su empleo lo consideran como una tradición familiar; además la grasa es empleada como ungüento para tratar quemaduras y heridas, ya que consideran que, al untar este aceite, su sabor y olor fuerte evita que lleguen las moscas y con ello las infecciones. Únicamente el $5 \%(n=2)$ de las personas mencionó el uso de la carne con fines medicinales, atribuyéndole un uso general contra cualquier enfermedad.

\section{Percepción}

Las palabras con las que los entrevistados relacionan al cocodrilo de pantano son: peligro, miedo, respeto, pantano, cinturón y cartera. El 95\% $(n=38)$ considera que el tamaño de la población actual es mayor a la que existía 10 años antes, y las causas del aumento es por las actuales restricciones de cacería y vigilancia de las autoridades para la protección de la especie. El $87.5 \%(n=35)$ de las personas considera que el cocodrilo de pantano es una especie importante dentro de la RBPC, ya que es un atractivo turístico, ayudan a mantener el nivel del agua en lagunas, ríos y el pantano y ayudan al buen funcionamiento del ecosistema. Los factores que las personas mencionaron afectan a las poblaciones de cocodrilo son la contaminación $(80 \%, n=32)$, quemas agrícolas $(50 \%, n=20)$, sequía $(22.5 \%, n=9)$, sobrepoblación $(20 \%, n=8)$ y cacería $(20 \%, n=8)$.

\section{Talleres de educación ambiental}

El número de asistentes a los talleres fue de 182 niños entre las tres comunidades. El 100\% ( $n=$ 182) tenían conocimiento sobre el cocodrilo de pantano, ya sea porque lo habían visto en el río o laguna, o bien, porque lo han comido. El total de niños dibujó 
al cocodrilo sin ayuda de algún facilitador, en todos los dibujos el cocodrilo fue representado dentro, o a la orilla de algún río o laguna y acompañado de otras especies, las más representativas fueron peces y tortugas (Figura 2a). De manera general, el cocodrilo de pantano es considerado por los niños como un animal peligroso al que le tienen respeto. Sin embargo, para ellos representa una fuente importante de alimento, mencionando que son animales con un sabor muy rico, que es consumido con frecuencia, al igual que otras especies silvestres de su región, entre ellas todos los tipos de tortugas, ardilla, venado, armadillo, mapache, mono, e iguana, principalmente y en menor proporción mencionaron al manatí. La mayoría de los niños mencionó que los avistamientos de cocodrilos es algo común para ellos, y que han visto desde crías hasta adultos, la mayoría de las veces estas interacciones se dan cuando acompañan a sus padres en la pesca. No consideran que exista algún problema por la presencia de estos animales y no tienen conocimiento de algún ataque en su comunidad.

Mediante plática y juegos (Figura $2 \mathrm{~b}$ y $2 \mathrm{c}$ ) se informó sobre las acciones benéficas que el cocodrilo de pantano realiza al ecosistema y se les preguntó a los niños sobre las actividades antropogénicas que consideran pueden amenazar las poblaciones de cocodrilos. Las respuestas más significativas fueron: matarlos para usarlos como alimento, la cacería, contaminación y quemas. Dentro de las actividades del taller, se presentó una obra de teatro interactiva (Figura 2d), en la que se integró la participación de los niños; al inicio de la obra, los niños mostraron alto interés por la cacería y consumo del cocodrilo; sin embargo, conforme avanzó el acto teatral y se explicaron los beneficios que este animal provee al ecosistema y los daños que se ocasionan al consumirlo, los niños hicieron compromisos para defenderlos y ayudar en su conservación.

\section{DISCUSIÓN}

\section{Conocimiento}

El conocimiento que las personas tienen sobre el cocodrilo de pantano es amplio y se basa principalmente en observaciones realizadas durante actividades como la pesca. Debido a que es una especie con la que conviven frecuentemente, identifican sus características morfológicas y de comportamiento, tienen conocimiento respecto a sus hábitos de alimentación, hábitat y épocas reproductivas. EI conocimiento de las personas que viven dentro de áreas naturales protegidas del cocodrilo ha sido documentado por Rodas-Trejo et al. (2018) en el Área de Protección de Flora y Fauna Laguna de Términos (APFFLT) en Campeche México, Balaguera-Reina y González-Maya (2010) en el Parque Nacional Vía Parque Isla Salamanca dentro del Caribe Colombiano, Padilla y Perera-Trejo (2010) en la Reserva de la Biósfera Los Petenes, Campeche, México. Este conocimiento se atribuye a la cercanía y frecuencia de avistamientos del cocodrilo durante las actividades diarias. De manera contraria, García-Grajales y Buenrostro (2015) identificaron que los habitantes de las comunidades aledañas al Parque Nacional Lagunas de Chacagua en Oaxaca, México, tienen un evidente desconocimiento sobre aspectos biológicos del cocodrilo americano, aun cuando la mayoría realiza sus actividades cotidianas en cercana relación con el ambiente natural y los cocodrilos.

\section{Interacciones}

En varios estudios, las interacciones hombrecocodrilo identifican un conflicto entre esas especies (Balaguera-Reina y González-Maya 2010, PeñaMondragón et al. 2013, Aranda-Coello et al. 2015), por los daños que ocasionan por consumo de peces, ruptura de redes de pesca, pérdida de animales de granja por depredación y principalmente ataques a personas (Cupul-Magaña et al. 2010, Wallace et al. 2012). Sin embargo, los entrevistados de la RBPC comentaron que no consideran que exista problema con la especie ya que no recuerdan algún ataque a personas dentro de su localidad, ni tampoco pérdidas de animales domésticos a causa de ataques por cocodrilos. Al respecto, RodríguezCalderón et al. (2018) en un trabajo sobre la relación entre productores agropecuarios rurales y la fauna silvestre de comunidades de Tabasco, reportan varias especies que las personas consideran dañinas sin 


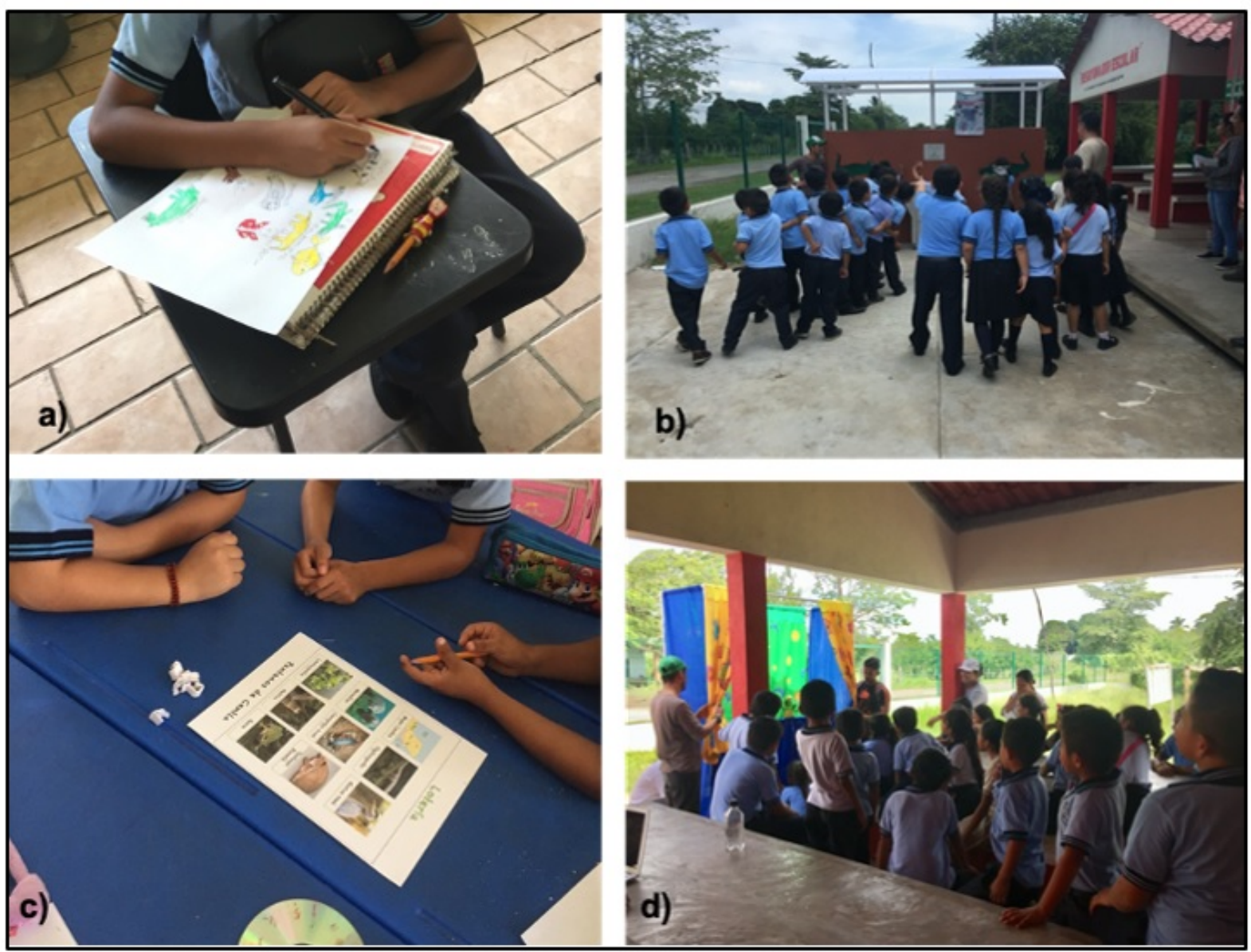

Figura 2. Actividades realizadas durante los talleres de educación ambiental. a) Dibujo representando a la fauna de la localidad. b) Juego. c) Lotería. d) Obra de teatro con títeres.

que el cocodrilo de pantano se encuentre reportado como depredador. Sin embargo, en el trabajo realizado por Pérez-Sánchez et al. (2006) en comunidades de la RBPC se vislumbra un conflicto hombre-cocodrilo, ya que el $40 \%$ de los entrevistados consideró al cocodrilo de pantano como una especie mala, principalmente por representar una amenaza para niños, atacar a las personas y comerse a los animales domésticos.

\section{Usos y aprovechamiento}

El uso y aprovechamiento del cocodrilo ha sido ampliamente documentado como se muestra en la Tabla 3. Años atrás, la comercialización de pieles para la elaboración de zapatos, cinturones, carteras y otros artículos fue una actividad que puso en riesgo las poblaciones de cocodrilos de América, ya que estos productos eran muy cotizados en el comercio europeo, a tal grado que, entre las décadas de 1930 y 1940, un comerciante de Villahermosa, Tabasco, podía llegar a vender más de 500 pieles en un solo día (Sigler y Gallegos, 2017). No obstante, a partir de 1970 con la veda permanente de las tres especies de cocodrilos que existen en México, el comercio ilegal de pieles dejó de ser una actividad redituable en las comunidades, ya que, con las restricciones legales, el precio pagado por las pieles es tan bajo, que no se compensa el peligro al que las personas se exponen durante la cacería.

Otro uso importante que se le ha dado al cocodrilo, es el de alimento, Balaguera-Reina y González- 
Tabla 3. Usos y aprovechamiento del Cocodrilo de pantano (Crocodylus moreletii). Al: Alimento, Me: Medicinal, C:Comercio, P:Peletero, Ma: Mascota, Ar:Artesanía, T:Turismo, Mi: Místico, F:Fotografía.

\begin{tabular}{|c|c|c|c|c|c|c|c|c|c|c|}
\hline Autor & Lugar & Al & $\mathrm{Me}$ & C & $\mathrm{P}$ & Ma & $\mathrm{Ar}$ & $\mathrm{T}$ & Mi & $\mathrm{F}$ \\
\hline Romeu (1998) & México & $\mathrm{X}$ & $\mathrm{X}$ & $\mathrm{X}$ & & & & & & \\
\hline Cupul-Magaña (2003) & Diferentes culturas del mundo & & $\mathrm{X}$ & & & & & & & \\
\hline Pérez-Sánchez et al. (2006) & RBPC, Tabasco, México & $\mathrm{X}$ & $\mathrm{X}$ & $\mathrm{X}$ & & & $\mathrm{X}$ & & & \\
\hline Arias (2007) & Tabasco, México & $x$ & $x$ & $x$ & & & & & & \\
\hline Da Nóbrega et al. (2008) & Diferentes culturas del mundo & & $\mathrm{X}$ & & & & & & & \\
\hline Balaguera-Reina y González-Maya (2010) & VIPIS, Colombia & $\mathrm{X}$ & & $\mathrm{X}$ & & & & & & \\
\hline Padilla y Perera-Trejo (2010) & RB Los Petenes, Campeche, México & $\mathrm{X}$ & $\mathrm{X}$ & $\mathrm{X}$ & & $\mathrm{X}$ & & & & \\
\hline González-Bocanegra et al. (2011) & $\begin{array}{l}\text { Los Humedales de Catazajá - La Liber- } \\
\text { tad, Chiapas, México }\end{array}$ & $x$ & $\mathrm{x}$ & $\mathrm{x}$ & & & $\mathrm{X}$ & & & \\
\hline Santos et al. (2011) & Tabasco, México & $\mathrm{X}$ & & & & & & & & \\
\hline Valdelomar et al. (2012) & ACT, Guanacaste, Costa Rica. & $x$ & $\mathrm{X}$ & & & $\mathrm{X}$ & $\mathrm{X}$ & $X$ & & \\
\hline Peña-Mondragón et al. (2013) & RB Chamela-Cuixmala, Jalisco, México & $\mathrm{X}$ & & & $\mathrm{X}$ & & & & $X$ & \\
\hline Aranda-Coello et al. (2015) & RNVSCN, Costa Rica & $x$ & & $X$ & & & & & & \\
\hline Muñoz y Santos (2015) & Tabasco, México & & & $x$ & & & & & & \\
\hline Contreras-Moreno (2018) & Tabasco, México & $\mathrm{X}$ & $\mathrm{X}$ & & & & & & & \\
\hline Lorente (2018) & Tabasco, México & $\mathrm{X}$ & & & & & & & & \\
\hline Rodas-Trejo et al. (2018) & APFFLT, Campeche, México & $\mathrm{X}$ & $\mathrm{X}$ & $\mathrm{X}$ & & & $\mathrm{X}$ & & & $X$ \\
\hline
\end{tabular}

Maya (2010) en Colombia, Peña-Mondragón et al. (2013) y Rodas-Trejo et al. (2018) en México, reportan que el $36 \%$ de los pobladores que viven dentro o en zonas cercanas a Reservas, consumen frecuentemente la carne de cocodrilo, lo cual se aproxima a los datos obtenidos en este estudio, que fue del $37.5 \%$. Específicamente en el estado de Tabasco, Lorente (2018) reporta que para los mayas chontales es común el aprovechamiento de la carne de cocodrilo, cocida, asada, frita o acompañada de plátano en caldo, en adobo, como relleno de tamales y empanadas, cocinada en guiso entomatado o con chile dulce; también Pérez-Sánchez et al. (2006), Arias (2007), Figueroa et al. (2000), Santos et al. (2011) reportan la preparación de diversos platillos con carne de cocodrilo en comunidades del estado de Tabasco. Desde tiempos antiguos y para diversas culturas como la Maya y Mexica, el cocodrilo se ha empleado como medicina para la curación del alma y el cuerpo, ya que desde distintas cosmovisiones se le atribuyen poderes terapéuticos (Cupul-Magaña 2003). En la actualidad, este uso continúa siendo aplicado por los habitantes de la RBPC, quienes refieren utilizarlo como remedio efectivo para tratar enfermedades respiratorias, curación de quemaduras y heridas tanto en las personas como en animales. Estos mismos usos fueron reportados por RodasTrejo et al. (2018) en el APFFLT, es muy probable que, al ser áreas naturales protegidas de estados ve- cinos, compartan usos, remedios y tradiciones. La utilización de la grasa por los mayas chontales de Tabasco ha sido descrita para diversas afecciones por Lorente (2018), quien menciona que lo utilizan en las heridas como cicatrizante, para tratar afecciones del ganado producidas por mordeduras de insectos 0 murciélagos, en forma de jarabe para curar el asma y en mujeres embarazadas para proteger al niño e incrementar la fuerza del feto. En comunidades rurales de los Humedales de Catazajá - La Libertad, Chiapas, la carne y grasa de cocodrilo se han empleado como analgésicos, antiinflamatorios y vigorizantes (González-Bocanegra et al. 2011).

\section{Percepción}

De manera general, se percibe una actitud positiva por parte de los habitantes de las comunidades hacia los cocodrilos, debido a que dentro de la Reserva se han realizado constantes capacitaciones y talleres de educación ambiental por parte de la Comisión Nacional de Áreas Naturales Protegidas (CONANP), quienes han dado a conocer los beneficios ecológicos de varias especies que viven dentro de la zona. Otra de las posibles razones de la percepción positiva, es que no han sido víctimas de algún ataque por parte de estos animales, y que el avistamiento de ellos es un atractivo turístico que provee de beneficio económico; esta visión en la que se considera al cocodrilo una atracción turís- 
tica ha sido reportada por Mandujano (2014) en el río Grijalva en Chiapas, México, Abadía (2002) en el río Grande de Tárcoles en Costa Rica y Valdelomar et al. (2012) en el Área de Conservación Tempisque (ACT) en Guanacaste, Costa Rica. Uno de los factores mencionados por los entrevistados de la RBPC que preocupa y afecta considerablemente a las poblaciones de cocodrilos y otras especies de animales acuáticos que viven dentro de la Reserva es la contaminación del agua, tanto por pesticidas utilizados en el sector agropecuario como por sustancias derivadas de la industria petrolera, que consideran ha contribuido con la contaminación y el deterioro ecológico, lo cual ha sido reportado previamente por Pinkus-Rendón y Contreras-Sánchez (2012). Todos los habitantes mencionaron que recientemente se habían presentado varias muertes de manatíes (Trichechus manatus) principalmente en el área conocida como Los Bitzales, ubicada dentro de la RBPC, aunque también encontraron peces muertos en los afluentes de agua de su comunidad y una persona mencionó que encontró un cocodrilo muerto. Al momento de las entrevistas, esta situación tenía fuertemente preocupados a los habitantes, ya que consideraban que la pesca se podría ver afectada e incluso su propia salud al consumir animales provenientes de aguas contaminadas. Al respecto, Hidalgo et al. (2019) publicaron un trabajo referente a lo mencionado por las personas entrevistadas, en el que informaron de la presencia de 48 cadáveres de manatíes (Trichechus manatus) entre los meses de mayo y octubre de 2018 en Los Bitzales. Mediante diversos estudios y análisis identificaron las posibles causas de las muertes, entre ellas sugirieron un proceso multifactorial influenciado por la prolongada temporada de sequía en el estado, las altas temperaturas, la presencia de un alto contenido de coliformes fecales, patógenos, floración de algas nocivas y toxicidad de sustancias químicas derivadas de agroquímicos e hidrocarburos. En años anteriores, Díaz-González et al. (1995) analizaron la presencia de hidrocarburos aromáticos policíclicos en suelos ubicados en los márgenes de los ríos Bitzal y Usumacinta que atraviesan la RBPC, identificando la presencia de sustancias con propiedades car- cinogénicas, sugiriendo que la contaminación de esta zona tiene como principal fuente a las actividades petroleras de la región. También, Córdova-Carrillo et al. (2010) realizaron un trabajo en el que identificaron el uso de 14 tipos de agroquímicos en actividades agropecuarias dentro de la RBPC, sugiriendo que pueden estar afectando de manera indirecta a las poblaciones de cocodrilo de pantano. La presencia de residuos tóxicos derivados de la agricultura y ganadería ha sido documentada en cocodrilos en diversas partes del mundo, encontrándolos a diferentes concentraciones y en varias partes del cuerpo como cartílagos, músculo, grasa, huevos y sangre presentando toxicidades de medias a muy altas (González et al. 2012, Sherwin et al. 2016). En muchos casos la Organización Mundial de la Salud (OMS) los clasifica como extremadamente peligrosos, ya que, aquellos animales que no mueren por los efectos de los contaminantes, pueden ser causantes de un envenenamiento secundario, es decir, causar la muerte de un depredador por ingerir presas contaminadas (Lazo-Serrano, 2017). Esta situación se convierte en un riesgo importante a la salud pública por prácticas como el consumo de carne y grasa de cocodrilo, ya sea como alimento o remedio medicinal para curar algunas enfermedades.

\section{Talleres de educación ambiental}

Los niños son uno de los sectores de la población que demuestran interés por la conservación de las especies y son parte fundamental para lograr estos objetivos (Galli et al. 2013), por lo que, es importante tomar en cuenta e identificar el conocimiento, actitudes y percepción que tienen hacia el medioambiente (Páramo y Galvis, 2010), para integrar estrategias que coadyuven a la conservación de las especies. A través de la técnica del dibujo se obtuvo información del conocimiento de los niños hacia su medio natural y en específico hacia el cocodrilo de pantano, esta técnica ha sido utilizada por Barraza (1999) quien menciona que los dibujos de los niños son herramientas útiles para proporcionar información valiosa para la evaluación de las percepciones ambientales. Para los niños el consumo de animales silvestre es una actividad frecuente, in- 
cluyendo en su dieta principalmente mamíferos y reptiles, demostrando gran gusto por el sabor de estos animales, principalmente por todas las especies de tortugas, lo cual ha sido reportado en estados colindantes a Tabasco por Ocampo-González et al. (2018) en el APFFLT en Campeche, México y por GonzálezBocanegra et al. (2011) en Chiapas, México. La actitud que los niños demostraron hacia el cocodrilo de pantano antes de iniciar las actividades de educación ambiental fueron enfocadas hacia la cacería y consumo, y aunque mencionaron que es un animal al que perciben como agresivo y le tienen respeto, no consideran temerle, ya que los observan con mucha frecuencia y para ellos representa un platillo en la cocina tradicional familiar, junto con otras especies que viven dentro de la Reserva.

\section{CONCLUSIONES}

Las personas de las localidades tienen conocimiento sobre el cocodrilo de pantano, ya que interactúan con ellos en actividades diarias como la pesca. De manera general, se percibe una actitud positiva, ya que conocen los beneficios ecológicos que proporcionan, además de que no reconocen ataques a personas dentro en sus localidades y el avistamiento de ellos es un atractivo turístico que provee un beneficio económico. El uso y aprovechamiento de la especie se da en todas las localidades principalmente como alimento y medicina. El principal factor que preocupa a los pobladores en la conservación de la especie es la contaminación de los ríos y lagunas con sustancias tóxicas derivadas de la industria petrolera y actividades agropecuarias, ya que también pueden verse afectados al ser consumir de carne y grasa de animales contamina- dos. Por lo que es necesario realizar estudios sobre presencia de sustancias tóxicas en los animales y agua, también se requiere difundir los resultados de los estudios. Los adultos tienen conocimiento sobre la especie tanto de tipo biológico como de los servicios ecosistémicos que brindan, es necesario realizar actividades de educación ambiental con los niños, ya que ellos desconocen los beneficios que la especie provee, y básicamente lo identifican como alimento, además de estar a favor de la cacería de especies silvestres, incluyendo tortuga, iguana, cocodrilo, armadillo, tlacuache, mono e incluso manatí. De manera general, las acciones de educación ambiental realizadas han contribuido en la percepción positiva que se tiene en la RBPC hacia el cocodrilo de pantano. Pero es necesario reforzar acciones para la conservación de diversas especies silvestres tanto en niños como adultos, ya que su consumo es una actividad común en la Reserva.

\section{AGRADECIMIENTOS}

A la Dirección de la Reserva de la Biosfera Pantanos de Centla de la Comisión Nacional de Áreas Naturales Protegidas (CONANP) y al Programa de Conservación de Especies en Riesgo (PROCER) por el financiamiento otorgado. A las personas entrevistadas por sus conocimientos y experiencias, a los integrantes del Programa de Manejo de Áreas Protegidas (PROMANP) de La Mixteca Segunda, Lázaro Cárdenas (El Guao), Tabasquillo y Tembladeras, a los directores, profesores, padres de familia y alumnos de las Escuelas Primarias "Coronel Lino Merino" en Lázaro Cárdenas (El Guao), "Benito Juárez García" del Ejido Tembladera y "Veinte de Noviembre" en la Ribera Tabasquillo Primera Sección.

\section{LITERATURA CITADA}

Abadía G (2002) Redituabilidad ecoturística de los cocodrilos del Grande de Tárcoles. Revista de Ciencias Ambientales (Trop J Environ Sci) 24: 34-43.

Albuquerque UP, Ramos MA, Lucena RFP, Alencar NL (2014) Methods and techniques used to collect ethnobiological data. In: Albuquerque UP, Cunha LVFC, Lucena RFP, Alves RRN (Eds). Methods and techniques in ethnobiology and ethnoecology. Springer. New York, USA. pp: 15-37. 
Aranda-Coello JM, Arévalo-Hueso E, Burbano D, Coello H, Cortéz J, Díaz N, Guerra L, Guevara C, Gutiérrez D, loli G, Jiménez R, Lobos L, Narváez V, Rico-Urones A (2015) Opinión de pescadores sobre el Caiman crocodilus (Crocodilia: Alligatoridae) del Refugio de Vida Silvestre Caño Negro, Costa Rica. Cuadernos de Investigación UNED 7: 143-149.

Arias OTE (2007) El cocodrilo en la región mayaYokot'an: Un acercamiento antropológico a la actualidad del ambiente en Tabasco. Itinerarios 6: 101-122.

Balaguera-Reina SA, González-Maya JF (2010) Percepciones, conocimiento y relaciones entre los Crocodylia y poblaciones humanas en la Vía Parque Isla de Salamanca y su zona de amortiguamiento, Caribe colombiano. Revista Latinoamericana de Conservación 1: 53-63.

Barba ME, Valadez CF, Pinkus RMA, Pinkus RMJ (2014) Revisión de la problemática socioambiental de la Reserva de la Biósfera Pantanos de Centla, Tabasco. Investigación y Ciencia 60: 50-57.

Barraza L (1999) Children's drawings about the environment. Environmental Education Research 5: 49-66.

Bernard HR (1996) Research methods in anthropology: Qualitative and quantitative approaches. American Journal of Evaluation 17: 91-92.

Cedillo-Leal CN, Requena-Lara G, Martínez-González JC, Vázquez-Loya DA, Cienfuegos-Rivas EG (2019) Distribución de Crocodylus moreletii Dumeril \& Bibron en Tamaulipas, México. Agroproductividad 12: 59-64.

Contreras-Moreno FM (2018) Usos diversos de la fauna silvestre en Tabasco. La Gaceta. Instituto Tecnológico Superior de los Ríos 1: 12-17

Córdova-Carrillo A, Pérez-Sánchez E, Rodríguez-Quevedo F, Ovando-Hidalgo N, Zequeira-Larios C (2010) Agroquímicos utilizados en la Reserva de la Biosfera Pantanos de Centla: una afectación indirecta para el Cocodrilo de Pantano (Crocodylus moreletii). Kuxulkab' 17: 31-42.

Cupul-Magaña FG (2003) Cocodrilo: medicina para el alma y el cuerpo. Revista Biomedica 14: 45-48.

Cupul-Magaña FG, Rubio-Delgado A, Reyes-Núñez C, Torres- Campos E, Solís-Pecero LA (2010) Ataques de cocodrilo de río (Crocodylus acutus) en Puerto Vallarta, Jalisco, México: Presentación de cinco casos. Cuadernos de Medicina Forense 16: 153-60.

Da Nóbrega ARR, Da Silva VWL, Gomes SG (2008) Reptiles used in traditional folk medicine: conservation implications. Biodiversity and Conservation 17: 2037-2049.

Díaz-González J, Cámara-Córdova J, Botello VA, Ponce VG (1995) Evaluación de hidrocarburos aromáticos policíclicos en suelos hidromórficos de la Reserva de la Biósfera "Los Pantanos de Centla" en Tabasco, México. Revista Internacional de Contaminación Ambiental 12: 15-21.

Figueroa OB, Gómez GJE, Rodríguez VW, Méndez MC, Méndez HA, Rodríguez QF, Hernández SM, Maldonado RN (2000) Datos preliminares del monitoreo de poblaciones silvestres de Crocodylus moreletii en los municipios de Nacajuca, Jonuta y Balancán del estado de Tabasco, México. In: Crocodiles: Proc. 15th working meeting of the CSG. IUCN-The World Conservation Union Gland, Switzerland. pp: 314-317.

Galli F, Bolzan de CC, Bedin LM, Castellá JS (2013) Actitudes hacia el medio ambiente en la infancia: un análisis de niños del sur de Brasil. Revista Latinoamericana de Psicología 45: 461-473.

García-Grajales J (2013) El conflicto hombre-cocodrilo en México: causas e implicaciones. Interciencia 38: 881-884.

García-Grajales J, Buenrostro SA (2015) Nota científica: Apreciación local acerca del cocodrilo americano (Crocodylus acutus) en comunidades rurales del Parque Nacional Lagunas de Chacahua, (Oaxaca, México). Etnobiología 13: 73-80. 
González-Bocanegra K, Romero-Berny El, Escobar-Ocampo MC, García-Del Valle Y (2011) Aprovechamiento de fauna silvestre por comunidades rurales en los humedales de Catazajá-La Libertad, Chiapas, México. Ra Ximhai 7: 219-230.

González-Jáuregui M, Valdespino CA, Salame-Méndez, Aguirre-León G, Rendón-VonOsten J (2012) Persistent organic contaminants and steroid hormones levels in Morelet's crocodiles from the Southern Gulf of Mexico. Archives of Environmental Contamination and Toxicology 62: 445-454.

Guerra-Martínez V, Ochoa-Gaona S (2008) Evaluación del programa de manejo de la Reserva de la Biosfera Pantanos de Centla en Tabasco, México. Universidad y Ciencia 24: 135-146.

Hidalgo RRA, Gómez GAO, Arreola SMM (2019) Manatees Mortality Analysis at Los Bitzales, Tabasco, by Remote Sensing. International Journal of Latest Research in Engineering and Technology 5: 1-15

INE (2000) Programa de Manejo Reserva de la Biósfera Pantanos de Centla, México. Instituto Nacional de Ecología. México. 222p.

INE (2000) Programa de manejo de la reserva de la biosfera Pantanos de Centla, México. Instituto Nacional de Ecología. Distrito Federal, México. 222p. http://www.paot.mx/centro/ine-semarnat/anp/AN30.pdf. Fecha de consulta 22 de mayo de 2020.

INEGI (2015) Catálogo de claves de entidades federativas, municipios y localidades. Instituto Nacional de Estadística y Geografía http://geoweb.inegi.org.mx/mgn2k/catalogo.jsp. Fecha de consulta: 12 de mayo de 2020.

Lazo-Serrano E (2017) Efecto del uso de agroquímicos en vertebrados silvestres. Conference Proceedings UTMACH 1: 1140-1148.

Lorente FD (2018) Pejelagartos, cocodrilos y canoas. De los seres del agua bajo el dominio de Ix Bolon entre los mayas chontales de Tabasco. Anales de Antropología 52: 179-195.

Mandujano CH (2014) Cultura y actitud hacia el cocodrilo (Crocodylus acutus) por usufructuarios del río Grijalva en Chiapas, México. Quehacer Científico en Chiapas 9: 10-23.

Mandujano CH, Hénaut YL (2010) Eclosión prematura de Crocodylus moreletii por "Ilamados eclosionales". Revista Quehacer Científico en Chiapas 9: 28-33.

Muñoz CJM, Santos RAJ (2015) Personas de la tercera edad de comunidades rurales y la cocina tradicional de Tabasco, México. Revista Española de Nutrición Comunitaria 21: 29-33

Ocampo-González P, Rodas-Trejo J, Hernández-Nava J, Solís-Marroquín D, Chang-Gutierrez D (2018) Consumo cultural y percepción social hacia las tortugas dulceacuícolas en el Área de Protección de Flora y Fauna Laguna de Términos, Campeche, México. Agroproductividad 11: 60-65.

Padilla SE, Perera-Trejo E (2010) Anotaciones sobre la percepción del Cocodrilo de Pantano por las comunidades mayas aledañas a la Reserva de la Biosfera Los Petenes. Revista Latinoamericana de Conservación 1: 8390.

Páramo P, Galvis CJ (2010). Conceptualizaciones acerca de los animales en niños de la sociedad mayoritaria y de la comunidad indígena Uitoto en Colombia. Revista Folios 32: 111-124.

Peña-Mondragón JL, García A, Vega RJH, Castillo A (2013) Interacciones y percepciones sociales con cocodrilo de río (Crocodylus acutus) en la costa sur de Jalisco, México. Revista de Biodiversidad Neotropical 3: 37-41. 
Pérez-Sánchez E, Rodríguez QF, Zequeira LC, Cruz VJ, Ovando HN (2006) Las comunidades rurales del cocodrilo de pantano (Crocodylus moreletti) en la Reserva de la Biosfera Pantanos de Centla. Kuxulkab' 11: 29-34.

Pinkus-Rendón MJ, Contreras-Sánchez A (2012) Impacto socioambiental de la industria petrolera en Tabasco: el caso de la Chontalpa. LiminaR Estudios Sociales y Humanísticos 10: 122-144.

Rodríguez-Calderon YG, Contreras-Moreno FM, Segura-Berttolini EC, Bautista-Ramírez P, Jesús-Espinosa D (2018) Análisis del conflicto entre la fauna silvestre y productores rurales en dos comunidades de Balancán, Tabasco, México. Agroproductividad 11: 51-59.

Romero GJC, García MA, Bautista JCA, Pérez APH (2000) Caracterización de la Reserva de la Biósfera Pantanos de Centla. Universidad y Ciencia 15: 15-28.

Romeu E (1998) Cocodrilos Mexicanos. Biodiversitas 4: 2-8.

Rodas-Trejo J, Ocampo-González P, Hernández-Nava J, Mandujano-Camacho H, Coutiño-Hernández PR, Orantes-Zebadua MA (2018) Percepción, conocimiento popular y aprovechamiento hacia el cocodrilo de pantano (Crocodylus moreletii Dumeril \& Bibron) por pobladores del Área de Protección de Flora y Fauna Laguna de Términos, Campeche, México. Agroproductividad 11: 45-50.

Santos RAJ, Muñoz CJM, Córdova HJA (2011) Hábitos de abasto en familias rurales de Tabasco. En: Muñoz CJM (Coord.). Sobrepeso, obesidad y diabetes: Diversos enfoques para su estudio. Colección Julián Manzur Ocaña. Vida y Salud Social. Universidad Juárez Autónoma de Tabasco. México. 161p.

SEMARNAP (1999) Secretaría de Medio Ambiente, Recursos Naturales y Pesca Proyecto para la Conservación, Manejo y Aprovechamiento Sustentable de los Crocodylia en México (COMACROM). SEMARNAP. México. $107 p$.

Sherwin B, Mudge J, Cañas CJ, Lanza H, Rainwater T, Platt S, McMurry S y Anderson T (2016) Organochlorine Pesticide Residues in Caudal Scutes of Belize Morelet's Crocodiles (Crocodylus moreletii). Journal of Herpetology 50: 552-558.

Sigler L, Gallegos MJ (2017) El conocimiento sobre el cocodrilo de Morelet Crocodylus moreletii (Duméril y Duméril 1851) en México, Belice y Guatemala. The Dallas World Aquarium. México. 216p.

Valdelomar V, Ramírez-Vargas MA, Quesada-Acuña SG, Arrieta C, Carranza I, Ruiz-Morales G, EspinozaBolaños S, Mena-Villalobos JM, Brizuela C, Miranda- Fonseca L, Matarrita-Herrera M, González-Venegas J, Calderón-Sancho E, Araya JF, Sauma-Rossi A, Sandoval-Hernández I, Gómez-Lépiz A (2012) Percepción y conocimiento popular sobre el cocodrilo Crocodylus acutus (Reptilia: Crocodylidae) en zonas aledañas al río Tempisque, Guanacaste, Costa Rica. Cuadernos de Investigación UNED 4: 191-202.

Wallace KM, Leslie AJ, Coulson T (2012) Living with predators: A focus on the issues of human-crocodile conflict within the lower Zambezi valley. Wildlife Research 38: 747-55. 